\title{
A continuum mechanics analysis of shear characterisation methods
}

\author{
Remko Akkerman \\ University of Twente \& TPRC, Enschede, The Netherlands
}

\section{A R T I C L E I N F O}

\section{Keywords:}

A: Fabrics/textiles

C: Analytical modelling

D: Mechanical testing

E: Forming

\begin{abstract}
A B S T R A C T
The shear response of fabrics and fabric reinforced materials is primarily characterised by means of Picture Frame and Bias Extension experiments. Normalisation methods have been proposed earlier to enable comparison between different measurement results. Here, a continuum mechanics based analysis is presented for biaxial fabric materials without intra-ply slip, subject to constraints of fibre inextensibility. The fibre stresses are separated from the constitutively determined extra stresses, leading to a scalar equivalent stress resultant which makes the analysis of these tests more transparent. The equations are elaborated for both shear characterisation experiments and a direct measurement evaluation is proposed, without the need for iterative parameter identification methods.
\end{abstract}

\section{Introduction}

Composite forming simulations can be used for successful part design and process optimisation, provided the relevant deformation mechanisms are included in the simulations appropriately and the materials are properly characterised, such that the simulations make use of the right material property data. Forming composite laminates usually involves significant bending and inplane shear, in particular for fabric reinforcements. In light of this, shear behaviour of fabrics and fabric reinforced polymers has received considerable attention in the last decades. 2D woven fabrics are amongst the most used and researched fabrics in this respect, with the often cited Esaform benchmark as a prime example [1]. The two tests evaluated in this benchmark, Picture Frame (PF) and Bias Extension (BE), are the primary experiments currently used for shear characterisation of fabric materials, with BE appearing to be the most preferred option. Both methods have their advantages but also their limitations, in terms of control of the experimental boundary conditions (in particular the occurrence of undesirable fibre stresses for $\mathrm{PF}$ ) and in terms of homogeneity of the deformation field (BE specimens have zones of different shear deformation). Ideally, both tests would provide comparable basic material property data, independent of specimen dimensions. This has proven difficult to be achieved in general. The preferential approach in $\mathrm{BE}$ testing nowadays is to determine the external power from the applied force and clamp displacement rate and to iteratively determine the stress power contributions of the different regions in the specimen to this total power.

Continuum models have proven their use in defining invaluable concepts such as equivalent stresses, strains and strain rates, and related material constants such as yield stress, shear and elongational viscosities, which are commonly applied in material characterisation and selection. Such concepts are not in use for fabric reinforced plastics, which is remarkable when realising that these materials ideally have only a single inplane deformation degree of freedom, related to the relative angle between the two fibre directions (as long as bending and intraply slip are absent). However, the measured stress power versus deformation or deformation rate data cannot be translated simply and uniquely to a total stress as a function of deformation or deformation rate. The total stresses can be considered to be simply the sum of fibre stresses and structural/material related stresses, where the fibre stresses do not contribute to the stress power when there is no fibre extension. Nevertheless, they are expected to affect the resistance to shear deformation, probably in some nonlinear manner. However, the level of fibre stresses is unclear from the currently used evaluations of test results.

As it is not obvious what part of the actual stresses is carried by the fibres during testing, also the test results cannot easily be reduced to the constitutively determined material/structural related shear stress terms or a related material constant such as a modulus or a viscosity. Moreover, various authors [2-4] have been unsuccessful in attempting to get to a transparent unified interpretation of both tests and to prove the assumption that both tests measure essentially the same phenomenon of trellis shear with only a single inplane deformation degree of freedom under plane stress conditions. Launay et al. [5] achieve better agreement between the shear forces for PF and $\mathrm{BE}$ testing if the fibre tension in the picture frame is kept zero during the test. It is suggested that the fibre tension is negligible during $\mathrm{BE}$ testing as the yarn ends are free at the edge, which seems strange when considering that the

E-mail address: r.akkerman@utwente.nl. 
longitudinal force in the specimen can only be transferred by the fibres. Hivet and Duong [4] conclude that 'the bias extension and picture frame tests do not exactly impose the same loading on the sample, and therefore, the results obtained cannot be identical.' On the other hand Harrison [6] shows good predictive capabilities of a comprehensive method (including bending and torsion measurements) applying the normalisation method as summarised above.

In order to derive an analysis of the shear test results which is free from these spurious terms, this paper presents the continuum theory to separate the fibre stresses from the constitutively determined extra stresses, leading to a scalar equivalent stress resultant (different from the equivalent stress in [2]) in which these fibre stresses are excluded. It will be shown that this equivalent extra stress resultant fully captures the constitutively determined stress state as it governs all extra stress resultant components. This reduces the characterisation efforts to determining the relation between this equivalent stress resultant and the relevant state variables, such as shear deformation, deformation rate and fibre tension. The theory applies for arbitrary material behaviour, whether or not path or rate dependent. Depending on the observed material response, this can lead to viscosities or moduli, which may depend on state variables such as fibre tension, temperature and pressure.

The theory is applied for PF and BE experiments, leading to simple expressions to derive the relevant fibre and equivalent stress resultants from the local deformation and the applied load. By this means, the results of both tests can be compared and observed differences can be related directly to differences in the underlying state variables, e.g. due to the boundary conditions imposed.

\section{Previous work}

The mechanical behaviour of fabric materials has been subject of many scientific publications in the past century. Various reviews are available in the open literature on this subject area. Hu [7] presented a broad overview on characterisation and modelling techniques for the structural mechanics of fabric materials. Bassett et al. [8] reviewed the development of inplane fabric testing, whereas Boisse et al. [9] reviewed the issues encountered in bias extension testing and modelling, such as fibre bending and intraply slip. A limited subset of the earlier publications on the mechanical behaviour of fabric materials will be addressed briefly in the current context.

Early work in this field, related to the development of Zeppelin airships, dates back to 1912, later translated to English [10]. In the field of apparel fabrics, efforts were made to translate subjective expert's feel or 'handle' of textile materials to objective physical tests of which the results were subsequently elaborated to physical properties such as the flexural rigidity [11]. Some years later, Peirce presented the basis for mesomechanical analyses of plain weave structures [12]. In 1961, Kilby [13] noted that also the shear resistance affected 'fabric hand' which required to be further examined. An instrument inducing uniform shear, e.g. as introduced by Mörner and Eeg-Olofsson [14] could be used for this purpose, but also extension testing in the bias directions as described by Weissenberg [15] (presenting one of the earliest images of $\mathrm{BE}$ experiments) or in other directions different from the fibre direction [16]. The latter references already apply the concept of trellis deformation, adopting the deformations of 'two sets of parallel rigid rods, mutually pinpointed where they cross, and capable of extending and contracting in certain directions without a change in the length or the form of the rods'. Note that this is different to simple shear, where the normal strains remain zero during shearing.

Spivak and Treloar [2] compared the responses for trellis shear testing and bias extension. An equivalent shear strain and shear stress were introduced, where the latter includes possible fibre tension. This increasingly affects the results for bias extension with increasing shear, at least partially causing discrepancy between the equivalent stresses for both experiments at equal equivalent strains. The authors therefore preferred 'simple' shear experimentation (imposing trellis shear) and suggested energy loss as a better way to compare the two type of fabric shear experiments. Skelton [17] further analysed the origin of fabric shear stiffness in terms of friction between the yarns. The fabrics' maximum shear angle was found to be related to the fabric tightness. Skelton further noted that fabrics have no relevant out-of-plane dimension and hence advised against the use of thickness to define stresses, preferring the use of mesomechanical analyses considering the crossover points in particular.

Again in order to precisely define subjective 'handling' or 'feeling' of materials, Kawabata [18] presented a series of mechanical tests enabling objective measurements, which do need to be interpreted by 'the man who well knows about the mechanical properties relating with its end use'. The resulting Kawabata Evaluation System for Fabrics (KES-F) quickly became the standardised fabric characterisation system [7], containing a variety of fabric tests to be carried out. An analysis of the forces encountered during KES-F shear testing, relating the torque around the intersection points to the overall shear force, was presented in [19]. An international interlaboratory trial comparing the outcomes of KES-F measurements [20] from 1988 showed poor reproducibility between the labs.

Research on characterisation for composites forming purposes started around the same time. Early work on the rheology of fabric reinforced plastics concerned continuum modelling of viscous, viscoelastic and (elasto-) plastic materials, by Rogers [21], McGuinness and ÓBrádaigh [22] for both modelling and experimental characterisation, and a generic continuum theory for fabric-reinforced fluids by Spencer [23]. Later, especially mesomechanical approaches as introduced earlier $[17,19]$ were elaborated in detail by e.g. Hivet et al. $[4,24]$ to obtain a better understanding of the deformation behaviour of fabric materials, describing the fabric structure and studying the structural deformations during forming operations. The global loads on the material are expressed in terms of the loads and torques acting on the crossover points of the yarns, which can be elaborated to covariant and contravariant stress components in the two fibre directions. As can be expected, the fabric geometry with often non-orthogonal fibre orientations leads to fairly lengthy mathematical expressions. Lomov et al. [25] evaluated the deformations during PF testing on the mesoscale by means of optical strain field measurements, and concluded that fibre tension dominates the fabric's shear resistance. The spatial variation of shear deformation was found to be limited for these tests. In order to provide good control of the shear deformations in BE specimens, deformations in theoretically undeformable regions can be prevented by locally bonding aluminium foil to the fabric [6].

From the perspective of a macroscopic power balance, Harrison et al. [26,27] presented a normalisation method for PF and uni-axial BE experiments, applied for thermoplastic and thermoset matrix composites. A similar approach was used by Peng et al. [28] for dry fabrics, and taken further by Cao et al. [1] in the earlier cited international shear characterisation benchmark exercise. These approaches use the measured force - displacement data to determine a relation between the stress power and the shear angle, assuming the shear behaviour is rate independent. According to the theory, there are two different regions with different non-zero (unknown) stress powers and (known) different shear rates. A single solution can be found for the stress power versus the shear angle by means of an iterative procedure $[3,4,29,30]$, employing a constitutive equation for the shear stress as a function of the shear angle. Alternatively, specimens of different sizes can be used to achieve a similar result [31]. This stress power can be translated to an equivalent shear force per unit length, which is being related to the force imposed in the longitudinal direction of the BE specimen [27]. In this approach it is assumed that fibre tension does not contribute to the deformation energy as a consequence of the inextensibility constraint.

Here, an attempt is made to clarify the magnitude and the role of the fibre stresses in shear characterisation experiments. The material will be described as a homogeneous continuum on the macroscopic scale, 
where it can be considered to be in plane stress conditions. In order to circumvent the problems with the out-of-plane dimension [17], the membrane stress resultant force per unit width, in short 'stress resultant' will be used, instead of a load per unit cross sectional area, i.e. stress. A similar formalism was used by Kilby [32], proposing anisotropic elastic laminae to describe the material behaviour for small deformations and Hu's generic non-linear framework [7]. The stress resultant tensor $\tau$ is equal to the thickness integrated Cauchy stress and will be represented in 2D matrix components of an inplane orthonormal coordinate system,

$\tau=\left[\begin{array}{ll}\tau_{11} & \tau_{12} \\ \tau_{12} & \tau_{22}\end{array}\right]$

The power required to deform a unit area is equal to the double contraction of the stress resultant tensor and $\boldsymbol{D}$, the rate of deformation tensor,

$\phi_{A}=\tau: \boldsymbol{D}=\tau_{11} \cdot \dot{\varepsilon}_{11}+\tau_{12} \cdot \dot{\gamma}_{12}+\tau_{22} \cdot \dot{\varepsilon}_{22}$,

with $\dot{\varepsilon}$ and $\dot{\gamma}$ as the normal and shear strain rates, respectively. For plane stress conditions, any change in thickness does not contribute to the total power as the normal stress is zero.

\section{Trellis shear deformations}

First, we will analyse the geometry, strains, strain rates and fibre orientations during trellis shear. The resulting orientation tensors will be used to find a basis spanning the space of symmetric $2 \times 2 \mathrm{D}$ tensors, with which the fibre stresses can subsequently be separated from the constitutively determined material related stresses.

The displacements under trellis shear deformation can be described by considering the length and width of a rectangular piece of fabric, as illustrated in Fig. 1, with the sides of the rhombus indicating the fibre directions, under an angle of $\pm \varphi$ with the first bias direction $x_{1}$. This leads to

$L=2 S \cos \varphi$,

$W=2 S \sin \varphi$.

We will consider the cases where $L \geqslant W>0$, implying for the fibre angle $\frac{1}{4} \pi \geqslant \varphi>0$. The shear angle $\gamma$ is related to the fibre angle by means of

$\gamma=\frac{1}{2} \pi-2 \varphi$,

with $0 \leqslant \gamma<\frac{1}{2} \pi$. The trellis arm is considered to be inextensible, in other words the length $S$ remains constant under trellis shear deformation. This implies that the principal stretches are equal to

$\lambda_{I}=\frac{L}{L_{0}}=\frac{2 S \cos \varphi}{2 S \cos \varphi_{0}}=\frac{\cos \varphi}{\cos \varphi_{0}}$,

$\lambda_{I I}=\frac{W}{W_{0}}=\frac{2 S \sin \varphi}{2 S \sin \varphi_{0}}=\frac{\sin \varphi}{\sin \varphi_{0}}$,

where subscript 0 indicates the initial state and $I, I I$ indicate the principal directions. Corresponding to these inplane deformations, the thickness changes from $H_{0}$ to $H$, which can be largely left out of consideration in case of plane stress conditions. The bias directions coincide with the principal directions of strains and strain rates. We will use

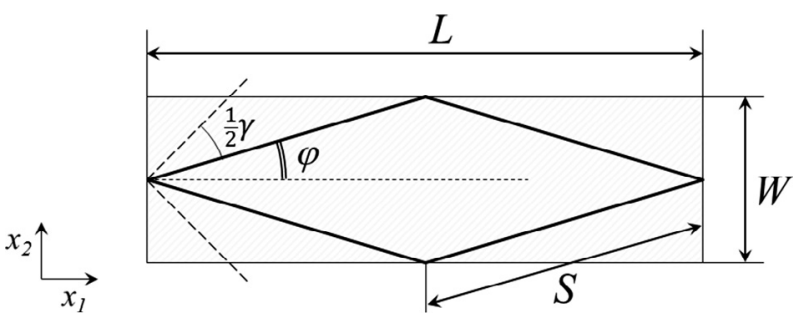

Fig. 1. Length and width of a repetitive fabric area under trellis shear deformation. this coordinate system in the remainder of this analysis. The principal strain rates $\dot{\varepsilon}_{I}$ and $\dot{\varepsilon}_{I I}$ can be derived easily from the rate of change of the dimensions of the rectangle in Fig. 1,

$\dot{\varepsilon}_{I}=\frac{\dot{L}}{L}=\frac{-\sin \varphi \cdot \dot{\varphi}}{\cos \varphi}=-\tan \varphi \cdot \dot{\varphi}$,

$\dot{\varepsilon}_{I I}=\frac{\dot{W}}{W}=\frac{\cos \varphi \cdot \dot{\varphi}}{\sin \varphi}=\frac{\dot{\varphi}}{\tan \varphi}$,

which leads to the deformation rate in the same $2 \mathrm{D}$ matrix representation

$[\boldsymbol{D}]=-\dot{\varphi}\left[\begin{array}{ll}\tan \varphi & 0 \\ 0 & -\frac{1}{\tan \varphi}\end{array}\right]$.

Now define the second order fibre orientation tensor $\boldsymbol{A}_{i}$ as the dyadic product of the unitary fibre orientation vectors $\boldsymbol{a}_{i}$,

$\boldsymbol{A}_{i}=\boldsymbol{a}_{i} \boldsymbol{a}_{i}$

The planar unitary orientation vectors and tensors are given by

$\boldsymbol{a}_{1}=\left[\begin{array}{l}\cos \varphi \\ \sin \varphi\end{array}\right] \Rightarrow \boldsymbol{A}_{1}=\left[\begin{array}{ll}\cos ^{2} \varphi & \sin \varphi \cos \varphi \\ \sin \varphi \cos \varphi & \sin ^{2} \varphi\end{array}\right]$

$\boldsymbol{a}_{2}=\left[\begin{array}{l}\cos \varphi \\ -\sin \varphi\end{array}\right] \Rightarrow \boldsymbol{A}_{2}=\left[\begin{array}{ll}\cos ^{2} \varphi & -\sin \varphi \cos \varphi \\ -\sin \varphi \cos \varphi & \sin ^{2} \varphi\end{array}\right]$

For non-zero shear deformations these orientation tensors are no longer orthogonal, as

$\boldsymbol{A}_{1}: \boldsymbol{A}_{2}=\cos ^{4} \varphi-2 \sin ^{2} \varphi \cos ^{2} \varphi+\sin ^{4} \varphi=\left(\cos ^{2} \varphi-\sin ^{2} \varphi\right)^{2}=\cos ^{2} 2 \varphi$,

which is non-zero for $\varphi \neq \frac{1}{4} \pi$. The tensors $\boldsymbol{A}_{1}$ and $\boldsymbol{A}_{2}$ will not coincide as long as $\varphi>0$. In order to span the space of symmetric $2 \times 2 D$ tensors we first observe that the tensor

$\boldsymbol{Z}=\left[\begin{array}{cc}\sin ^{2} \varphi & 0 \\ 0 & -\cos ^{2} \varphi\end{array}\right]$

is orthogonal to $\boldsymbol{A}_{1}$ and $\boldsymbol{A}_{2}$, as $\boldsymbol{A}_{1}: \boldsymbol{Z}=\boldsymbol{A}_{2}: \boldsymbol{Z}=0$. We define the third unitary tensor $\boldsymbol{A}_{3}$ as

$\boldsymbol{A}_{3}=\frac{1}{\|\boldsymbol{Z}\|_{2}} \boldsymbol{Z}=\frac{1}{\sqrt{\sin ^{4} \varphi+\cos ^{4} \varphi}}\left[\begin{array}{cc}\sin ^{2} \varphi & 0 \\ 0 & -\cos ^{2} \varphi\end{array}\right]$.

Now, any symmetric $2 \times 2 D$ tensor $\boldsymbol{T}$ can be written as a linear combination of $\left\{\boldsymbol{A}_{1}, \boldsymbol{A}_{2}, \boldsymbol{A}_{3}\right\}$ according to

$\boldsymbol{T}=\lambda \boldsymbol{A}_{1}+\mu \boldsymbol{A}_{2}+\kappa \boldsymbol{A}_{3}$,

with

$\boldsymbol{T}: \boldsymbol{A}_{1}=\lambda+\mu \cos ^{2} 2 \varphi$,

$\boldsymbol{T}: \boldsymbol{A}_{2}=\lambda \cos ^{2} 2 \varphi+\mu$,

$T: A_{3}=\kappa$.

\section{Analysis of stress resultant forces}

The stress resultant tensor will now be split into parts depending on the constraints and a part depending on the constitutive behaviour only. To this end, both the rate of deformation and the stress resultant tensors will be expressed in terms of the basis $\left\{\boldsymbol{A}_{1}, \boldsymbol{A}_{2}, \boldsymbol{A}_{3}\right\}$. All unknown scalar components ( $\lambda, \mu, \kappa$ in Eq. (13)) can be determined by elaborating the stress power and the constraints. As a result, the fibre stress resultants can be separated from the constitutively determined material response.

First split the rate of deformation according to (13),

$\boldsymbol{D}=\lambda_{D} \boldsymbol{A}_{1}+\mu_{D} \boldsymbol{A}_{2}+\kappa_{D} \boldsymbol{A}_{3}$.

As trellis shear induces zero strain in the fibre directions, i.e. 
$\boldsymbol{D}: \boldsymbol{A}_{i}=0, \quad(i=1,2)$,

it directly follows that $\lambda_{D}=\mu_{D}=0$. Using (7), (12) and (14) results in

$\kappa_{D}=\boldsymbol{D}: \boldsymbol{A}_{3}$

$=\frac{-\dot{\varphi}}{\sqrt{\sin ^{4} \varphi+\cos ^{4} \varphi}}\left(\tan \varphi \cdot \sin ^{2} \varphi+\frac{1}{\tan \varphi} \cdot \cos ^{2} \varphi\right)$

$=\frac{-\dot{\varphi} \sqrt{\sin ^{4} \varphi+\cos ^{4} \varphi}}{\sin \varphi \cos \varphi}$.

The stress resultant is split similarly, according to (13),

$\tau=\lambda_{\tau} \boldsymbol{A}_{1}+\mu_{\tau} \boldsymbol{A}_{2}+\kappa_{\tau} \boldsymbol{A}_{3}$.

The inextensibility constraints imply that there is no contribution to the power dissipation in the fibre directions. The stress resultants in these directions have the magnitudes

$\mathscr{T}_{i}=\tau: \boldsymbol{A}_{i}, \quad(i=1,2)$.

Applying (14) implies for these fibre stress resultants $\mathscr{T}_{i}$ that

$\mathscr{T}_{1}=\lambda_{\tau}+\mu_{\tau} \cos ^{2} 2 \varphi$,

$\mathscr{T}_{2}=\lambda_{\tau} \cos ^{2} 2 \varphi+\mu_{\tau}$.

Inverting this system of equations leads to an expression for the first two scalar variables in (18) as a function of the fibre stress resultants,

$\lambda_{\tau}=\frac{\mathscr{T}_{1}-\mathscr{T}_{2} \cos ^{2} 2 \varphi}{1-\cos ^{4} 2 \varphi}$,

$\mu_{\tau}=\frac{\mathscr{T}_{2}-\mathscr{T}_{1} \cos ^{2} 2 \varphi}{1-\cos ^{4} 2 \varphi}$.

The third constant, $\kappa_{\tau}$, can be resolved by means of the dissipative power, given by

$\phi_{A}=\tau: D$,

which, knowing that $\boldsymbol{A}_{3}$ is orthogonal to $\boldsymbol{A}_{1}$ and $\boldsymbol{A}_{2}$, can be elaborated to

$\phi_{A}=\left(\lambda_{D} \lambda_{\tau}\right) \boldsymbol{A}_{1}: \boldsymbol{A}_{1}+\left(\lambda_{D} \mu_{\tau}+\mu_{D} \lambda_{\tau}\right) \boldsymbol{A}_{1}: \boldsymbol{A}_{2}$

$+\left(\mu_{D} \mu_{\tau}\right) \boldsymbol{A}_{2}: \boldsymbol{A}_{2}+\left(\kappa_{D} \kappa_{\tau}\right) \boldsymbol{A}_{3}: \boldsymbol{A}_{3}=$

$=\kappa_{D} \kappa_{\tau}$.

With (17) this leads to

$\kappa_{\tau}=\frac{\phi_{A}}{\kappa_{D}}=\frac{-\phi_{A} \sin \varphi \cos \varphi}{\dot{\varphi} \sqrt{\sin ^{4} \varphi+\cos ^{4} \varphi}}$.

For the sake of convenience, we introduce an equivalent stress resultant according to

$\tilde{\tau}=\frac{\phi_{A}}{\dot{\gamma}}=-\frac{\phi_{A}}{2 \dot{\varphi}}$,

which, when substituted in (24), leads to

$\kappa_{\tau}=\tilde{\tau} \cdot \frac{2 \sin \varphi \cos \varphi}{\sqrt{\sin ^{4} \varphi+\cos ^{4} \varphi}}$.

Combining the results of the foregoing analysis leads to the following expression for the stress resultant tensor

$\tau=\lambda_{\tau} A_{1}+\mu_{\tau} A_{2}+\kappa_{\tau} A_{3} \equiv \tau_{1}^{f}+\tau_{2}^{f}+\tau^{m}$,

with

$\tau_{1}^{f}=\frac{\mathscr{T}_{1}-\mathscr{T}_{2} \cos ^{2} 2 \varphi}{1-\cos ^{4} 2 \varphi}\left[\begin{array}{cc}\cos ^{2} \varphi & \sin \varphi \cos \varphi \\ \sin \varphi \cos \varphi & \sin ^{2} \varphi\end{array}\right]$,

$\tau_{2}^{f}=\frac{\mathscr{T}_{2}-\mathscr{T}_{1} \cos ^{2} 2 \varphi}{1-\cos ^{4} 2 \varphi}\left[\begin{array}{cc}\cos ^{2} \varphi & -\sin \varphi \cos \varphi \\ -\sin \varphi \cos \varphi & \sin ^{2} \varphi\end{array}\right]$,

$\tau^{m}=\frac{\tilde{\tau} \cdot 2 \sin \varphi \cos \varphi}{\left(\sin ^{4} \varphi+\cos ^{4} \varphi\right)}\left[\begin{array}{cc}\sin ^{2} \varphi & 0 \\ 0 & -\cos ^{2} \varphi\end{array}\right]$,

or, in terms of the shear angle $\gamma$, which is a more intuitive measure for deformation in a constitutive model,
$\tau_{1}^{f}=\frac{\mathscr{T}_{1}-\mathscr{T}_{2} \sin ^{2} \gamma}{2\left(1-\sin ^{4} \gamma\right)}\left[\begin{array}{cc}1+\sin \gamma & \cos \gamma \\ \cos \gamma & 1-\sin \gamma\end{array}\right]$

$\tau_{2}^{f}=\frac{\mathscr{T}_{2}-\mathscr{T}_{1} \sin ^{2} \gamma}{2\left(1-\sin ^{4} \gamma\right)}\left[\begin{array}{cc}1+\sin \gamma & -\cos \gamma \\ -\cos \gamma & 1-\sin \gamma\end{array}\right]$,

$\tau^{m}=\frac{\tilde{\tau} \cos \gamma}{\left(1+\sin ^{2} \gamma\right)}\left[\begin{array}{cc}1-\sin \gamma & 0 \\ 0 & -1-\sin \gamma\end{array}\right]$.

The equations for the stress resultants in the fibre direction $\tau_{1,2}^{f}$ contain a priori unknown scalars $\mathscr{T}_{1}$ and $\mathscr{T}_{2}$ which cannot be resolved constitutively i.e. through the deformation history. These terms can only be resolved by equilibrium considerations in relation to the externally applied forces. As formulated by Truesdell and Noll [33] (using the current symbol $\boldsymbol{a}$ and deformation gradient $\boldsymbol{F}$ ) 'for materials that are inextensible in the direction of $\boldsymbol{a}$ in the reference configuration, the stress is determined by the deformation history only to within a uniaxial tension in the direction of $\boldsymbol{F} \cdot \boldsymbol{a}^{\prime}$. In other words, it is sufficient to consider only $\tau^{m}$ in Eqs. (27)-(29) as a function of the material behaviour, which is to be denoted as the extra stress resultant tensor.

The expression for this extra stress resultant tensor is a most relevant result of this analysis, which is derived without any assumptions concerning the constitutive behaviour apart from those leading to trellis shear. It implies that the constitutive behaviour of a fabric material as defined here, with a give fibre angle, can be captured in only one single scalar: the equivalent stress resultant. This scalar may well be a function of multiple state variables such as shear angle, shear rate, fibre tensions, compaction stress and temperature, and may be written in terms of multiple constants. This reduces the study of the shear behaviour of these materials to the study of the change of this scalar property with respect to the relevant state variables. Material characterisation experiments should deliver the equivalent stress resultant as a function of the selected state variables.

The selected constitutive model needs to be consistent with the presented expression of the extra stress resultant tensor. Its shear component is zero in the bias coordinate system and its normal components have a fixed ratio, as

$\tau_{22}^{m}=\frac{-\tau_{11}^{m}}{\tan ^{2} \varphi}$

The zero shear component of the extra stress resultant implies that the principal directions of the extra stress resultant tensor coincide with the bias directions too. Note that only when both fibre stress resultants are equal, $\mathscr{T}_{1}=\mathscr{T}_{2}$, this also applies for the total stress resultant.

By means of equilibrium considerations, the intra-ply shear stress resultants (related to possible intra-ply slip) can be expressed as the gradients of the fibre tensions in the respective fibre directions. In case of intra-ply slip, further material characterisation is needed to relate these intra-ply shear stress resultants to the slip and/or slip velocity vector (and other state variables). This is beyond the scope of the current elaboration.

\section{Alternative expressions for the equivalent stress resultant}

For the plane stress conditions assumed here, the accompanying stress power per unit area is given by (2) in an arbitrary orthonormal inplane coordinate system. As shown previously, the bias directions coincide with the principal directions of the strain rate and of the extra stress resultant, whereas the fibre stress resultants $\tau_{1}^{f}$ and $\tau_{2}^{f}$ (which may have different principal directions) do not contribute to the stress power. Expressed in the bias directions, the equation for the stress power per unit area reduces to

$\phi_{A}=\tau_{I}^{m} \cdot \dot{\varepsilon}_{I}+\tau_{I I}^{m} \cdot \dot{\varepsilon}_{I I}$,

with the principal strain rates related by (6). Consequently, under trellis shear conditions, 
$\phi_{A}=\left(\tau_{I}^{m}-\frac{\tau_{I I}^{m}}{\tan ^{2} \varphi}\right) \cdot \dot{\varepsilon}_{I}=\frac{1}{2}\left(\tau_{I}^{m} \tan \varphi-\frac{\tau_{I I}^{m}}{\tan \varphi}\right) \cdot \dot{\gamma}_{0}$,

implying that the equivalent stress resultant (25) is equal to

$\widetilde{\tau}=\frac{1}{2}\left(\tau_{I}^{m} \tan \varphi-\frac{\tau_{I I}^{m}}{\tan \varphi}\right)$.

Examining (28), it can be observed that the equivalent stress resultant can also be expressed directly in terms of the total stress resultant, when written in the bias directions, as

$\tilde{\tau}=\frac{1}{2}\left(\tau_{11} \tan \varphi-\frac{\tau_{22}}{\tan \varphi}\right)$.

A slightly simpler expression follows when transforming the Cauchy stress $\sigma$ to the 2nd Piola Kirchhoff (PK2) stress $\boldsymbol{S}$, related by

$\boldsymbol{S}=|\operatorname{det} \boldsymbol{F}| \boldsymbol{F}^{-1} \cdot \sigma \cdot \boldsymbol{F}^{-T}$.

Integrating the stress components over the thickness ( $H$ and $H_{0}$ for the current and original configuration, respectively) leads to the stress resultants in the original configuration (PK2 stress resultants)

$\boldsymbol{T}=\left[\begin{array}{ll}T_{11} & T_{12} \\ T_{12} & T_{22}\end{array}\right]=\left[\begin{array}{cc}\tau_{11} \tan \varphi & \tau_{12} \\ \tau_{12} & \frac{\tau_{22}}{\tan \varphi}\end{array}\right]$.

Splitting the PK2 stress resultant tensor into three terms as in (27) leads to

$\boldsymbol{T}_{1}^{f}=\left(\mathscr{T}_{1}-\mathscr{T}_{2} \cos ^{2} 2 \varphi\right) \frac{\sin \varphi \cos \varphi}{1-\cos ^{4} 2 \varphi}\left[\begin{array}{ll}1 & 1 \\ 1 & 1\end{array}\right]$,

$\boldsymbol{T}_{2}^{f}=\left(\mathscr{T}_{2}-\mathscr{T}_{1} \cos ^{2} 2 \varphi\right) \frac{\sin \varphi \cos \varphi}{1-\cos ^{4} 2 \varphi}\left[\begin{array}{cc}1 & -1 \\ -1 & 1\end{array}\right]$,

$\boldsymbol{T}^{m}=\frac{2 \tau}{\left(\sin ^{4} \varphi+\cos ^{4} \varphi\right)}\left[\begin{array}{cc}\sin ^{4} \varphi & 0 \\ 0 & -\cos ^{4} \varphi\end{array}\right]$,

implying that (33) can be rewritten in terms of the PK2 stress resultants as

$\widetilde{\tau}=\frac{1}{2}\left(T_{11}-T_{22}\right)=\frac{1}{2}\left(T_{11}^{m}-T_{22}^{m}\right)=\frac{1}{2}\left(T_{I}^{m}-T_{I I}^{m}\right)$.

This shows the equivalent stress resultant to be similar to the maximum shear stress as used in Tresca's yield criterion in solid mechanics. It can be graphically represented by the radius of the corresponding Mohr's circle of the extra stress resultants or indeed the maximum shear component of the extra stress resultant, but now expressed with respect to the undeformed configuration.

\section{Shear characterisation experiments}

The formalism introduced here will be applied to PF and BE experiments, leading to simple expressions for the fibre and equivalent stress resultants in terms of the external load and the major shear angle.

\subsection{Picture frame test}

The PF experimental set-up is schematically illustrated in Fig. 2, showing the arms of the frame of length $S$, the current length $L$, fibre angle $\varphi$, external force $F$ and cross head velocity $\dot{u}$.

For this geometry, the shear rate can be found to be

$\dot{\gamma}=-2 \dot{\varphi}=\frac{\dot{u}}{S \sin \varphi}$

The area of the tested material is given by

$A=S^{2} \sin 2 \varphi$

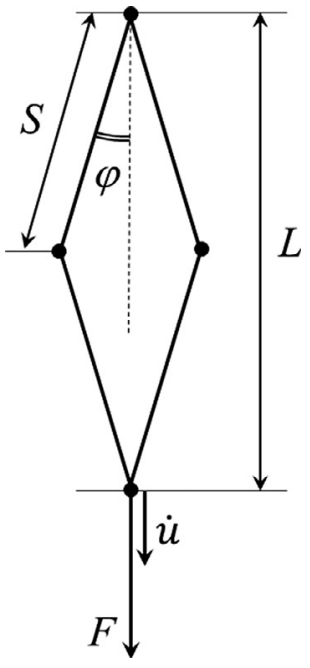

Fig. 2. Picture Frame geometry.

The external force is in equilibrium with the tensile forces $F_{L}$ built up in the legs of the fixture,

$F=2 \cos \varphi \cdot F_{L}$

which, in turn, are equal to the integrated shear stress resultant along the leg,

$F_{L}=\tau_{0} S$,

implying that no tension is transmitted through the free hinges of the frame. Thus, the external force translates to a shear stress resultant on the fabric material according to

$\tau_{0}=\frac{F}{2 S \cos \varphi}$.

The PF loading conditions are illustrated in Fig. 3(a), schematically showing a representative area of a sheared biaxially reinforced material deformed as a pin-jointed net, with shear angle $\gamma$ and $\varphi=\frac{1}{4} \pi-\frac{1}{2} \gamma$ as the fibre angle with respect to the bias direction $I$ in tension.

This figure further illustrates the envisaged fabric stress resultants. Only inplane shear stress resultants act on the fabric edges. The inplane normal stress resultants are supposed to be zero on these edges, in order to avoid fibre tension during testing as this will significantly increase the resultant force on the picture frame. Specific methods of clamping the fabric along the edges have been devised to deal with this issue, e.g. by using thin rubber sheet placed between the fabric and clamps or by allowing the lower clamps of the frame to slide in the normal direction. This fully defines the planar load situation under ideal PF conditions. The common tensor rotation formulae apply, by which the normal and shear stress resultants in any orientation can be derived by, respectively

$\tau_{n}(\theta)=\frac{1}{2}\left(\tau_{I}+\tau_{I I}\right)+\cos 2 \theta \cdot \frac{1}{2}\left(\tau_{I}-\tau_{I I}\right)$,

$\tau_{s}(\theta)=\sin 2 \theta \cdot \frac{1}{2}\left(\tau_{I}-\tau_{I I}\right)$,

with $\theta$ as the rotation angle with respect to the first principal direction and principal stress resultants $\tau_{I}, \tau_{I I}$. The fibre tensions are zero under ideal PF conditions, such that $\tau^{m}=\tau$. Hence, the principal stress resultant directions coincide with the bias directions (indicated with $I$ and II in Fig. 3). The maximum shear stress resultants are only initially (when $\gamma=0$ ) oriented along the edges of the representative area in Fig. 3(a). Mohr's circle in Fig. 3(b) provides a graphical representation of these rotation equations.

This circle can be derived mathematically but can also be 

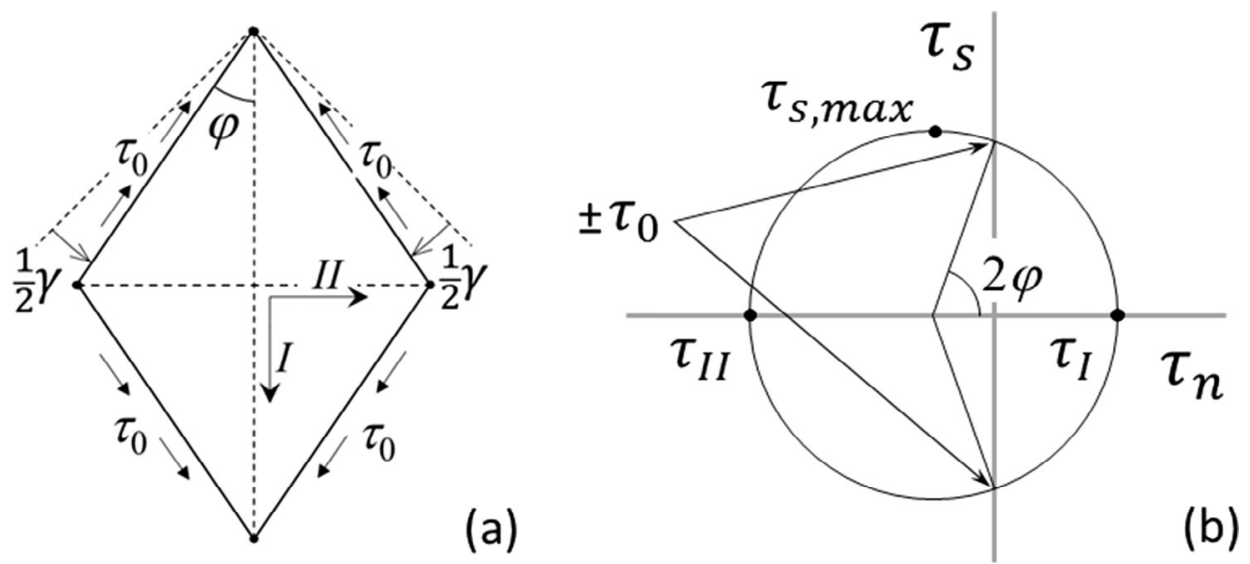

Fig. 3. Picture Frame experiment: (a) schematic loading conditions; (b) Mohr's circle for the normal and shear stress resultants.

constructed geometrically, by noting that the normal stress resultants are zero at the edges of the skewed element in Fig. 3(a), which leads to the intersections of the circle with the $\tau_{s}$ axis. The orientations of both edges differ by an amount of $2 \varphi$. Hence the angular positions of both points on Mohr's circle with respect to its midpoint differ by an amount of $2 \times 2 \varphi$, which is sufficient to complete the geometric construction as illustrated in Fig. 3(b). The PF loading condition thus leads to tension in direction $I$ and compression in direction $I I$, with the principal stress resultants given by

$\tau_{I}=\frac{\tau_{0}}{\sin 2 \varphi} \cdot(1-\cos 2 \varphi)=\tau_{0} \tan \varphi$,

$\tau_{I I}=-\frac{\tau_{0}}{\sin 2 \varphi} \cdot(1+\cos 2 \varphi)=\frac{-\tau_{0}}{\tan \varphi}$.

The circle moves further to the left with increasing shear. The transverse compression in the II direction may lead to buckling for decreasing fibre angles. Note that the fibre tensions, following from (19), remain zero throughout the test as long as the normal stresses induced by the fixture remain zero, even if these normal stresses have a direction different from the fibres. The maximum shear stress resultant differs increasingly from the exerted shear stress resultant $\tau_{0}$ :

$\tau_{s, \max }=\frac{\tau_{0}}{\sin 2 \varphi}$.

It can be seen that the components of the extra stress resultant, equal to the total stress resultant as argued above of which the principal components were specified in (45), satisfy (30). Via the analysis of stress resultant in this section, the equivalent stress resultant can be derived by substituting the terms in (33). A simpler way to reach the same results is by equating the area integrated specific stress power to the externally applied power. This directly leads to an expression for the equivalent stress resultant in terms of the external force $F$ and the fibre angle $\varphi$ for the current state of the material (angle, shear rate, temperature, pressure, etc.), after substitution of (25), (39) and (40):

$\Phi=A \cdot \phi_{A}=F \cdot \dot{u} \Rightarrow \tilde{\tau}=\frac{F}{2 S \cos \varphi}$,

and, by means of (28) and (29) or (37), to an expression for all components of the extra stress resultant tensor. Stress power and stress equilibrium represented by Mohr's circle lead to identical results for this case with uniform deformation.

\subsection{Bias Extension test}

Geometric AnalysisA BE specimen is a rectangular strip with the fibres oriented under $\pm \frac{\pi}{4}$ with the longitudinal axis, with initial width $W_{0}$, initial length between the clamps $L_{0}$ and length to width ratio $\Lambda=L_{0} / W_{0}$ with $\Lambda \geqslant 2$. Three regions can be distinguished during extension, as illustrated in Fig. 4, each with a different state of

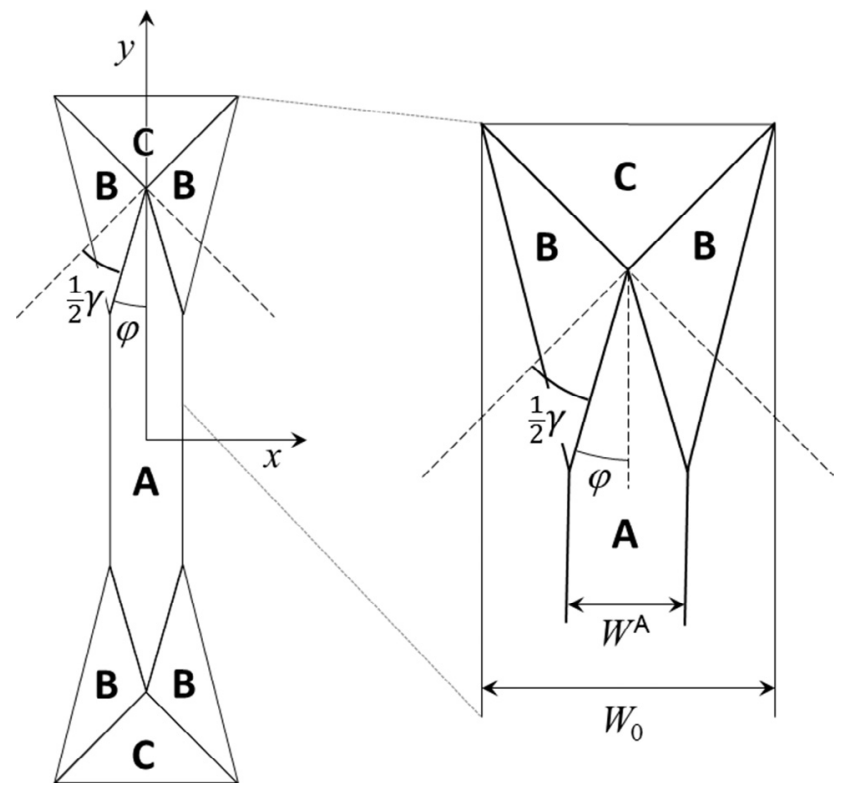

Fig. 4. Schematic representation of a deformed Bias Extension specimen. Left: the full free length of the specimen between the clamps, right: zoomed in with the width definitions $W^{\mathrm{A}}$ and $W_{0}$.

deformation which is supposed to be uniform per region.

In the pin-jointed apporach, neglecting fibre strain and intraply slip between the fibres, the outer region C in Fig. 4 remains undeformed. The clamp displacement $u$, equal to the elongation of the central region A, is related to the central fibre angle $\varphi$ with respect to the longitudinal axis of the specimen by

$u=(\Lambda-1) W_{0} \cdot(\sqrt{2} \cos \varphi-1)$.

The shear rate in the central zone is hence given by

$\dot{\gamma}=-2 \dot{\varphi}=\frac{\sqrt{2}}{(\Lambda-1) \sin \varphi} \frac{\dot{u}}{W_{0}}$.

The width of the central region of the specimen is related to the fibre angle by means of

$W^{\mathrm{A}}=W_{0} \sqrt{2} \sin \varphi$.

Fig. 5 shows the original and the deformed geometry of region B, from which it easily follows that

$2 \alpha+\frac{1}{2} \pi+\frac{1}{2} \gamma=\pi \Rightarrow \alpha=\frac{1}{4} \pi-\frac{1}{4} \gamma=\frac{1}{8} \pi+\frac{1}{2} \varphi$.

The angle between the fibres in region B increases with half of the 


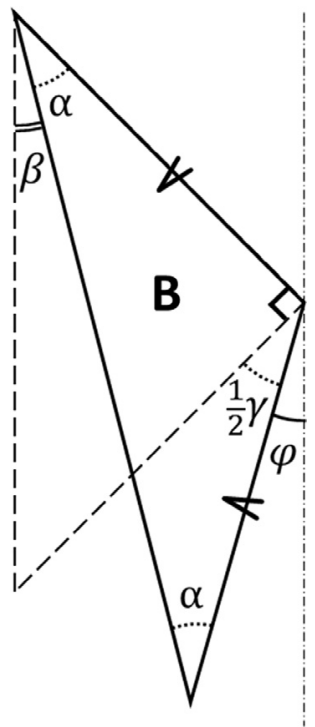

Fig. 5. Original (dashed lines) and deformed (solid lines) state of region B in a Bias Extension specimen.

shear angle in region $\mathrm{A}$, while the bias directions rotate to an angle $\beta=\frac{1}{4} \pi-\alpha=\frac{1}{4} \gamma$ with respect to the longitudinal axis of the specimen. The shear angle and the shear rate in region $\mathrm{B}$ are hence half of the corresponding values in region A.

The initial areas of the three regions can be found to be

$A_{0}^{\mathrm{A}}=\left(L_{0}-\frac{3}{2} W_{0}\right) W_{0}=\left(\Lambda-\frac{3}{2}\right) W_{0}^{2}$,

$A_{0}^{\mathrm{B}}=W_{0}^{2}$,

$A_{0}^{\mathrm{C}}=\frac{1}{2} W_{0}^{2}$,

which change upon extension according to

$A^{\mathrm{A}}=A_{0}^{\mathrm{A}} \sin 2 \varphi$,

$A^{\mathrm{B}}=A_{0}^{\mathrm{B}} \sin 2 \alpha=A_{0}^{\mathrm{B}} \cdot \frac{1}{2} \sqrt{2}(\cos \varphi+\sin \varphi)$.

Stress Resultants Being able to separate the fibre stresses from the constitutively determined response, it now makes sense to elaborate force equilibrium in addition the scalar power equilibrium. Quasi-static Finite Element (FE) simulation results should satisfy both, but the analytical normalisation methods cited earlier do not evaluate force equilibrium explicitly. It will be shown that this can lead to a direct method, making the use of iterative methods redundant.

In order to estimate the magnitude of the stress resultants in a $\mathrm{BE}$ specimen, it is assumed that the state of stress resultant is uniform in each of the three regions. In equilibrium, each cross section along the

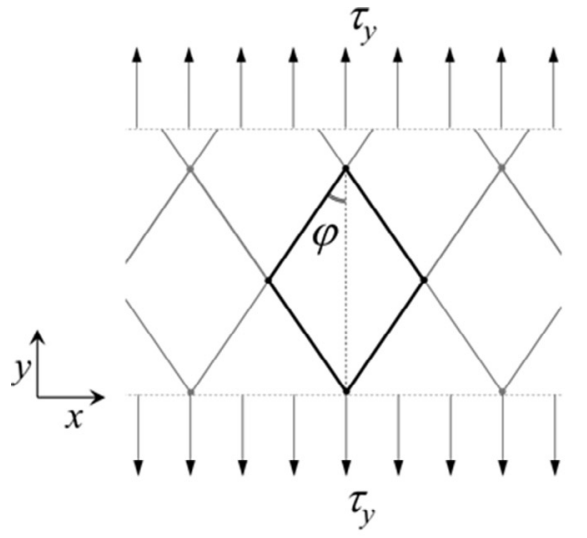

(a) length of the specimen transfers the same axial load $F$, equal to the integral of the normal stress resultant in $y$ direction over the local width $W(y)$,

$F_{y}=\int_{x=-\frac{1}{2} W(y)}^{\frac{1}{2} W(y)} \tau_{y}(x) \mathrm{d} x$,

to which possibly fibre tensions on the region boundaries of can be added, if these coincide with a fibre direction. The fibres constituting these boundaries will build up tension $\left(T_{f}\right)$ if the shear stress resultant is discontinuous over these boundaries, according to

$\frac{d T_{f}}{d s}=\Delta \tau_{s n}$

with $s, n$ as the co-ordinates in and normal to the fibre direction, respectively, and $\Delta \tau_{s n}$ as the difference in shear stress resultants on both sides of the boundary.

No such tensions are present on the free edge of the central region A when $\Lambda>2$. Hence the normal stress resultant in the $y$ direction must satisfy

$\tau_{y}^{\mathrm{A}}=\frac{F}{W^{\mathrm{A}}}=\frac{1}{\sqrt{2} \sin \varphi} \frac{F}{W_{0}}$.

Strictly, this expression is equivalent to the conventional definition of stress being equal to the force divided by the cross sectional area, but now for a specimen subject to trellis shear kinematics. The normal stress resultant in the $x$ direction is obviously zero in the central region, as well as the shear stress resultant $\tau_{x y}^{\mathrm{A}}$. This implies that region A is subjected to uniaxial tension, with principal stress resultants

$\tau_{I}^{\mathrm{A}}=\tau_{y}^{\mathrm{A}}, \tau_{I I}^{\mathrm{A}}=0$,

acting in the $y$ and $x$ direction, respectively. Fig. 6 illustrates the loading conditions and shows Mohr's circle for the stress resultants. Obviously the normal stress resultants are now tensile or zero in any direction (in contrast to the PF stress state depicted in Fig. 3, which is partially tensile and increasingly compressive as the test proceeds). The equivalent stress resultant of a region in uniaxial stress in the first bias direction follows from (34)

$\tilde{\tau}=\frac{1}{2} \tau_{11} \tan \varphi$.

For region A this leads to

$\widetilde{\tau}^{\mathrm{A}}=\frac{1}{2} \tau_{y}^{\mathrm{A}} \tan \varphi=\frac{\sqrt{2}}{4 \cos \varphi} \cdot \frac{F}{W_{0}}$.

It can be noted that this expression for the equivalent stress resultant is very similar to the 'Method 3' or the 'simple' equation in [29], which follows when $\Lambda \rightarrow \infty$ such that the effect of region B is negligible. In particular in the case of uniform deformation of a specimen

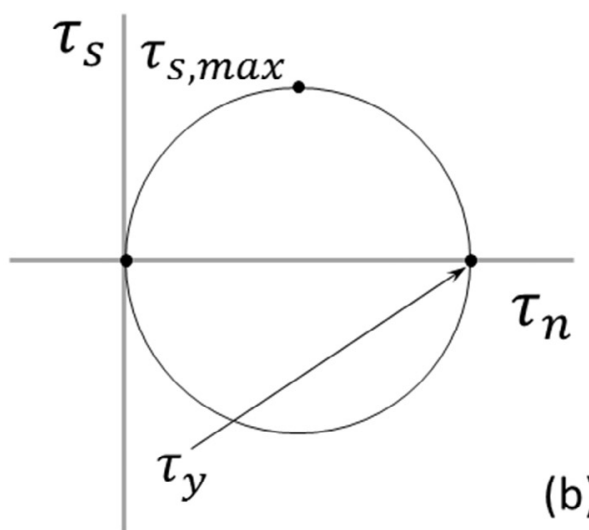

Fig. 6. Bias Extension experiment: (a) schematic loading conditions; (b) Mohr's circle for the normal and shear stress resultants. 
with a constant cross section along the length, force and power equilibrium yield the same solution. For smaller values of $\Lambda$ both equilibrium equations can lead to different expressions, which will be addressed later in more detail.

Elaborating (19) for this state of stress leads to the corresponding fibre stress resultants in region A

$\mathscr{T}_{1}=\mathscr{T}_{2}=\frac{\cos ^{2} \varphi}{\sqrt{2} \sin \varphi} \cdot \frac{F}{W_{0}}$.

The results above can be used to also derive an expression for the equivalent stress resultant in region $\mathrm{B}$, starting from the total stress power consumed by the specimen, which is equal to

$\Phi=A^{\mathrm{A}} \phi_{A}^{\mathrm{A}}+A^{\mathrm{B}} \phi_{A}^{\mathrm{B}}$,

as the deformations and stress power in region $\mathrm{C}$ are zero. With the previous definitions this can be elaborated to

$F \dot{u}=\left(\Lambda-\frac{3}{2}\right) W_{0}^{2} \sin 2 \varphi \cdot \widetilde{\tau}^{\mathrm{A}} \dot{\gamma}+W_{0}^{2} \cdot \frac{1}{2} \sqrt{2}(\cos \varphi+\sin \varphi) \cdot \widetilde{\tau}^{\mathrm{B}} \frac{1}{2} \dot{\gamma}$.

With (49) we find

$(\Lambda-1) \sqrt{2} \sin \varphi \cdot \frac{F}{W_{0}}=(2 \Lambda-3) \sin 2 \varphi \cdot \widetilde{\tau}^{\mathrm{A}}+\frac{1}{2} \sqrt{2}(\cos \varphi+\sin \varphi) \cdot \widetilde{\tau}^{\mathrm{B}}$,

which, although written in different symbols, is identical to the corrected relation (2) derived in [30] for the external force, indicating that Harisson's shear force per unit length $\left(F_{s} h\right)$ is equal to the equivalent stress resultant derived here (25). Substitution of (59) leads to

$\sin \varphi \cdot \frac{F}{W_{0}}=(\cos \varphi+\sin \varphi) \cdot \widetilde{\tau}^{\mathrm{B}}$,

such that the equivalent stress resultant in region B for shear angle $\gamma^{\mathrm{B}}=\frac{1}{4} \pi-\varphi$ is given by

$\widetilde{\tau}^{\mathrm{B}}=\frac{\sin \varphi}{\cos \varphi+\sin \varphi} \frac{F}{W_{0}}$.

The stress resultants derived here can be made dimensionless according to

$\tau^{*}=\frac{W_{0}}{F} \cdot \tau$.

The equivalent stress resultants in A and B (59) and (65) are plotted in their dimensionless form in Fig. 7 with respect to their local shear angle, $\gamma^{\mathrm{A}}$ and $\gamma^{\mathrm{B}}$ respectively, providing a compact representation of the current solution. Note, that the difference between both curves does not automatically imply that there is no unique relation between the shear angle and the equivalent stress resultant, as the loads for a given shear angle are different for both curves. In addition, the figure shows the fibre stress resultants in region A, which increasingly bear the tensile load as the shear angle increases, whereas the relative equivalent stress

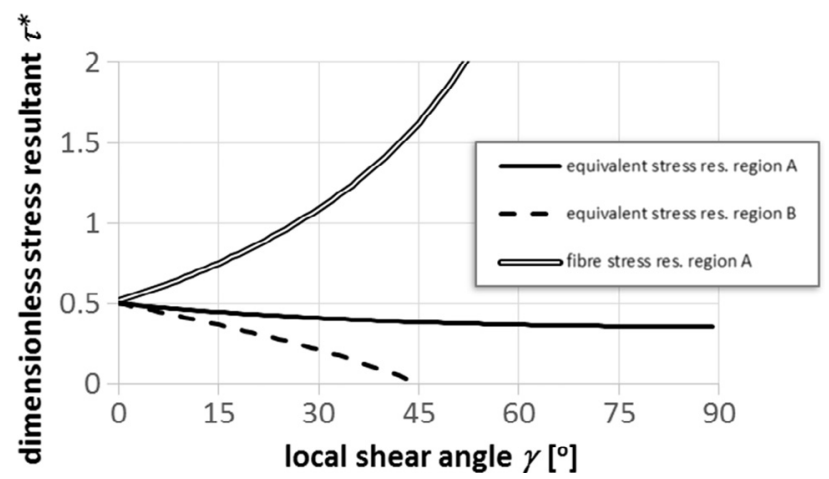

Fig. 7. Dimensionless stress resultants $\left(\tau^{*}=\frac{W_{0}}{F} \cdot \tau\right)$ in regions A and B of a Bias Extension specimen as a function of their local shear angle. resultants are gradually decreasing. This shows that the longitudinal force during $\mathrm{BE}$ is increasingly being carried by the fibres as the test proceeds.

It is possible to derive the local stress resultant components in regions $\mathrm{B}$ and $\mathrm{C}$ by further elaborating the current results. For the purpose of material characterisation, however, it suffices to measure the load and the displacement of the clamps, preferably supplemented with the shear angle and width of the central region A. This information leads to the stress resultants $\tau_{y}^{\mathrm{A}}$ and $\widetilde{\tau}^{\mathrm{A}}$ by means of (56) and (59), the fibre stress resultants by means of (60), the shear angle $\gamma$ and the shear rate $\dot{\gamma}$. The shear angle is preferably measured directly, as using (49) from the clamp displacement data is a known source of scatter in the experimental data $[3,4]$.

Thus, by having a closed form solution for the stress resultants in the $\mathrm{BE}$ specimen, combined with the split of the stress resultant tensor in fibre stress resultants and the constitutively determined extra stress resultant, it is possible to bypass the earlier mentioned iterative normalisation methods. These can be analytical for rate independent materials but often employ parameter identification methods requiring iterative FE analyses for more complex material behaviour as observed in e.g. thermoplastic composites in melt conditions to determine a 'best fit' of the material property data. Using the 'gage method' [3] with this new analysis provides a more transparent direct approach to the shear characterisation of fabric materials. The data analysis is illustrated in the spreadsheet accompanying this paper, providing a worked example of a bias extension measurement on a dry carbon fabric.Verification

The question rises how the current analysis compares to other approaches. First of all, FE simulations were performed using the implicit Aniform solver (v.3.4.1 [34]), for (arbitrary) nonlinear rate dependent material behaviour on a specimen with length to width ratio $\Lambda=2$. After quick convergence to machine precision (as in [35], using the same algorithms), force and stress power equilibrium equations are satisfied. The FE results show homogeneous deformations per region and confirm the assumption of a uniaxial stress state in region A. Also quantitatively, the total, fibre and hence equivalent stresses are in accordance with respectively (56) and the subsequent Eqs. (60) and (59) for each time step i.e. axial force $F$ vs. $\varphi$ combination, supporting the validity of the proposed method.

Further, the current analysis can be compared to the earlier cited iterative procedures e.g. $[3,4,30]$. All approaches start from the same basic assumptions: uniform deformations per region, no inter-tow slippage and inextensibility in the two fibre directions, with the same initial and boundary conditions. The earlier iterative approach starts from stress power equilibrium whereas the current analysis is based on force equilibrium and can also accomodate power equilibrium by means of (65). It seems logical that the earlier and the current approach would lead to the same results when considering material behaviour that does not depend on shear rate and fibre tension. However, when starting from an arbitrary relation between the equivalent stress resultant and the shear angle such as elaborated in [29], it is found that both approaches lead to different results and seem to be conflicting, which throws doubt on the validity of at least one of both. To clarify this matter, the foregoing assumptions and equations are reconsidered.

For rate and fibre tension independent behaviour, the equivalent stress resultant is a function of the shear angle only and similarly the axial force, such that with (59)

$\tilde{\tau}^{\mathrm{A}}=\tilde{\tau}(\gamma)=\frac{\sqrt{2}}{4 \cos \varphi} \cdot \frac{F(\gamma)}{W_{0}}, \quad \tilde{\tau}^{\mathrm{B}}=\tilde{\tau}\left(\frac{1}{2} \gamma\right)=\frac{\sqrt{2}}{4 \cos \varphi} \cdot \frac{F\left(\frac{1}{2} \gamma\right)}{W_{0}}$

Substitution into the stress power Eq. (63) leads to

$F(\gamma)=\frac{\frac{1}{2} \sqrt{2}(\cos \varphi+\sin \varphi)}{2 \sin \varphi \cos \varphi} \cdot F\left(\frac{1}{2} \gamma\right)$

which is equivalent to 


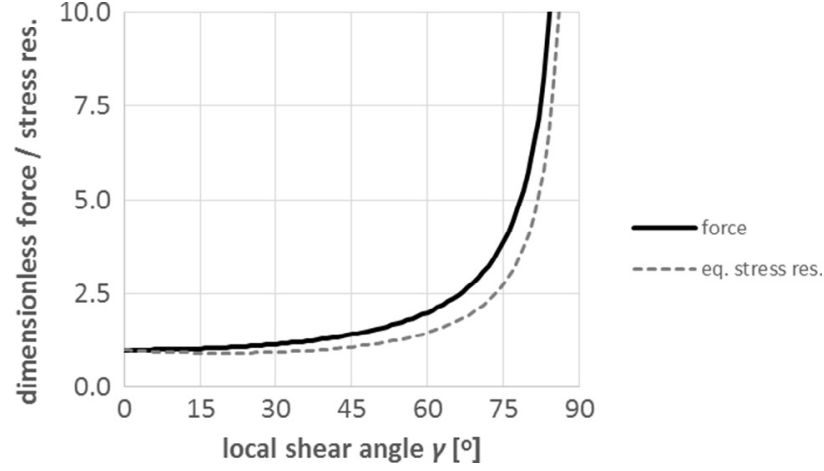

Fig. 8. Dimensionless axial force $\left(\frac{F(\gamma)}{c}\right.$, solid line) and equivalent stress resultant $\left(\frac{\tilde{\tau} W_{0}}{c}\right.$, dashed line) as a function of the local shear angle for rate and fibre stress independent shear behaviour.

$F(\gamma) \cdot \cos \gamma=F\left(\frac{1}{2} \gamma\right) \cdot \cos \frac{1}{2} \gamma$

The general continuous solution of this recursive transcendental equation is readily found by assuming (69) to be the same constant for all values of $\gamma$. Thus, when accepting (59) and (63) as the correct representations of force and power equilibrium and assuming that $\widetilde{\tau}$ is a function of $\gamma$ only, then the trellis shear kinematics require that the force versus shear angle relation must satisfy

$F(\gamma)=\frac{c}{\cos \gamma}$

with constant $c$. The corresponding equivalent stress resultant must be

$\tilde{\tau}(\gamma)=\frac{c}{2 W_{0}\left(\cos \frac{1}{2} \gamma+\sin \frac{1}{2} \gamma\right) \cos \gamma}$.

This force and equivalent stress resultant are plotted in dimensionless form in Fig. 8, showing curves quite similar to shear test results reported in the cited literature. Of course, the underlying assumptions of these curves are quite stringent and violations of these concerning intertow slip and fibre stress dependency have been addressed previously. Nevertheless, this characteristic shape of (70) is retained in practically all test results found in literature. Remarkably, the force is non-zero for zero shear (but also the velocity should be nonzero when considering power equilibrium). In experiments, the load at zero deformation would typically be set to zero at the start of the test to correct for clamp and specimen weight, so it is not trivial to draw any conclusions on this without going into further details.

The foregoing analysis implies, inversely, that when $F(\gamma) \cdot \cos \gamma$ is constant, then the underlying constitutive behaviour can be considered to be independent of shear rate and fibre tension. This condition is easily verified from measured force versus shear angle data. In other cases, the presumptions are conflicting. Considering that force and power equilibrium are generally accepted, that intertow slippage can be minimum up to moderate shear angles such that trellis shear kinematics are preserved, and that homogeneous deformations per region are observed in both experiments and FE simulations for at least a length to width ratio $\Lambda=2$, this leaves the independency of the shear force on shear rate or fibre tension as the most debatable underlying assumption of arbitrary shear force versus shear angle curves.

Finally, this comparison shows that the shear force per unit length $F_{s h}$ found from the iterative approach and which does not satisfy (71) cannot be used instead of $\widetilde{\tau}$ in the stress resultant Eq. (28) or (29). The equivalent stress resultant introduced here is easily determined from experiments, however, and independent of the actual constitutive behaviour. It is hence also applicable for material behaviour that does depend on shear rate or fibre tension, as observed in thermoset and thermoplastic composite prepregs.

\section{Conclusion}

An analysis was presented of the stresses and deformations in 2D fabric reinforced materials along the lines of classical continuum mechanics, using the 2D stress resultant tensor as a convenient means for this purpose. The extra stress resultant was identified, which separates the constitutively determined part of the stress resultant from the part related to the fibre stresses arising from the inextensibility constraints. It was shown that all extra stress resultant components can be expressed in terms of a scalar equivalent stress resultant, which thus fully captures the constitutive behaviour of such materials under trellis shear, in the absence of intra-ply slip. This analysis is independent of the actual material behaviour: whether or not depending on rate, fibre tension, or any other state variable. The resulting equations were elaborated for the two main shear characterisation methods, Picture Frame and Bias Extension experiments, leading to simple expressions for the major equivalent and fibre stress resultants (47), (59) and (60), summarised below:

\begin{tabular}{ccc}
\hline & Equivalent stress resultant & Fibre stress resultant \\
\hline $\mathrm{PF}$ & $\tilde{\tau}=\frac{1}{2 \cos \varphi} \cdot \frac{F}{S}$ & $\mathscr{T}=0$ (ideally) \\
$\mathrm{BE}$ & $\tilde{\tau}^{\mathrm{A}}=\frac{1}{2 \sqrt{2} \cos \varphi} \cdot \frac{F}{W_{0}}$ & $\mathscr{T}^{\mathrm{A}}=\frac{\cos ^{2} \varphi}{\sqrt{2} \sin \varphi} \cdot \frac{F}{W_{0}}$ \\
\hline
\end{tabular}

This work provides a convenient basis for the analysis of shear characterisation results for fabric materials, firstly in terms of the equivalent stress resultant as a function of the local state variables. These results can be employed to identify suitable constitutive models and subsequently to derive the appropriate material property data. Such a direct approach eliminates the need for parameter identification methods requiring iterative analyses to determine a 'best fit' of the material property data.

\section{Acknowledgements}

The author gratefully acknowledges the financial and technical support from the industrial and academic members of the ThermoPlastic composites Research Center (TPRC) as well as the support funding from the Province of Overijssel for improving the regional knowledge position within the Technology Base Twente initiative.

\section{Appendix A. Supplementary material}

Supplementary data associated with this article can be found, in the online version, at http://dx.doi.org/10.1016/j.compositesa.2018.02. 036.

\section{References}

[1] Cao J, Akkerman R, Boisse P, Chen J, Cheng HS, De Graaf EF, et al. Characterization of mechanical behavior of woven fabrics: experimental methods and benchmark results. Compos Part A: Appl Sci Manuf 2008;39(6):1037-53.

[2] Spivak SM, Treloar LRG. The behavior of fabrics in shear: Part III: the relation between bias extension and simple shear. Text Res J 1968;38(9):963-71.

[3] Harrison P, Wiggers J, Long AC. Normalization of shear test data for rate-independent compressible fabrics. J Compos Mater 2008;42(22):2315-44.

[4] Hivet G, Duong AV. A contribution to the analysis of the intrinsic shear behavior of fabrics. J Compos Mater 2011;45(6):695-716.

[5] Launay J, Hivet G, Duong AV, Boisse P. Experimental analysis of the influence of tensions on in plane shear behaviour of woven composite reinforcements. Compos Sci Technol 2008;68(2):506-15.

[6] Harrison P, Alvarez MF, Anderson D. Towards comprehensive characterisation and modelling of the forming and wrinkling mechanics of engineering fabrics. Int $\mathrm{J}$ Solids Struct. http://dx.doi.org/10.1016/j.ijsolstr.2016.11.008.

[7] $\mathrm{Hu}$ J. Structure and mechanics of woven fabrics. Woodhead Publishing Limited; 2004.

[8] Bassett RJ, Postle R, Pan N. Experimental methods for measuring fabric mechanical properties: a review and analysis. Text Res J 1999;69(11):866-75.

[9] Boisse P, Hamila N, Guzman-Maldonado E, Madeo A, Hivet G, Dellísola F. The bias- 
extension test for the analysis of in-plane shear properties of textile composite reinforcements and prepregs: a review. Int J Mater Form 2016:1-20.

[10] Haas R, Dietzius A. The Stretching of the Fabric and the Deformation of the Envelope in Nonrigid Balloons, Institute of Gas Technology, 1918. URL http://hdl. handle.net/2060/19930091043.

[11] Peirce FT. The handle of cloth as a measurable quantity. J Text Inst Trans 1930;21(9):T377-416.

[12] Peirce FT. 5 - The geometry of cloth structure. J Text Inst Trans 1937;28(3):T45-96.

[13] Kilby WF. Shear properties in relation to fabric hand. Text Res J 1961;31(1):72-3.

[14] Mörner B, Eeg-Olofsson T. Measurement of the shearing properties of fabrics. Text Res J 1957;27(8):611-5.

[15] Weissenberg K. 5 - The use of a trellis model in the mechanics of homogeneous materials. J Text Inst Trans 1949;40(2):T89-110.

[16] Chadwick GE, Shorter SA, Weissenberg K. 6 - A trellis model for the application and study of simple pulls in textile materials. J Text Inst Trans 1949;40(2):T111-60.

[17] Skelton J. Fundamentals of fabric shear. Text Res J 1976;46(12):862-9.

[18] Kawabata S. Analysis of handling criterion. Sen'i Gakkaishi 1969;25(5):191-9. http://dx.doi.org/10.2115/fiber.25.191.

[19] Kawabata S, Niwa M, Kawai H. 5 - The finite-deformation theory of plain-weave fabrics. Part III: the shear-deformation theory. J Text Inst 1973;64(2):62-85.

[20] Ly NG, Denby EF. A CSIRO inter-laboratory trial of the KES-F for measuring fabric properties. J Text Inst 1988;79(2):198-219.

[21] Rogers TG. Rheological characterization of anisotropic materials. Composites 1989;20(1):21-7.

[22] McGuinness GB, ÓBrádaigh CM. Development of rheological models for forming flows and picture-frame shear testing of fabric reinforced thermoplastic sheets. Non-Newton Fluid Mech 1997;73(1):1-28.

[23] Spencer AJM. Theory of fabric-reinforced viscous fluids. Compos Part A: Appl Sci Manuf 2000;31(12):1311-21.

[24] Hivet G, Vidal-Sallé E, Boisse P. Analysis of the stress components in a textile composite reinforcement. J Compos Mater 2013;47(3):269-85.

[25] Lomov SV, Willems A, Verpoest I, Zhu Y, Barburski M, Stoilova T. Picture frame test of woven composite reinforcements with a full-field strain registration. Text Res J 2006;76(3):243-52.

[26] P. Harrison, J. Wiggers, A. C. Long, Normalisation of shear test data for rate-independent compressible fabrics, in: 10th International ESAFORM Conference on Materials Forming, 18-20th April, Zaragoza, Spain, AIP, 2007, pp. 1011-1016.

[27] Harrison P, Clifford MJ, Long AC. Shear characterisation of viscous woven textile composites: a comparison between picture frame and bias extension experiments. Compos Sci Technol 2004;64(10):1453-65.

[28] Peng XQ, Cao J, Chen J, Xue P, Lussier DS, Liu L. Experimental and numerical analysis on normalization of picture frame tests for composite materials. Compos Sci Technol 2004;64(1):11-21.

[29] Härtel F, Harrison P. Evaluation of normalisation methods for uniaxial bias extension tests on engineering fabrics. Compos Part A: Appl Sci Manuf 2014;67:61-9.

[30] Harrison P, Härtel F. Corrigendum to 'evaluation of normalisation methods for uniaxial bias extension tests on engineering fabrics'. Compos Part A: Appl Sci Manuf 2016;80:104-6.

[31] Komeili M, Milani AS. An elaboration on the shear characterization of dry woven fabrics using trellising tests. Polym Compos 2013;34(3):359-67.

[32] Kilby WF. 2 - Planar stress-strain relationships in woven fabrics. J Text Inst Trans 1963;54(1):T9-27.

[33] C. Truesdell, W. Noll, The non-linear field theories of mechanics, in: Encyclopedia of Physics, Vol. 3/3, Springer, 1965.

[34] AniForm Engineering B.V., Aniform. URL https://www.aniform.com, accessed at: 2017-07-25.

[35] Ten Thije RHW, Akkerman R, Huétink J. Large deformation simulation of anisotropic material using an updated lagrangian finite element method. Comput Methods Appl Mech Eng 2007;196(33-34):3141-50. 